\title{
Hydrodynamic limit of multi-chain driven diffusive models
}

\author{
V. Popkov \\ Institut für Festkörperforschung, Forschungszentrum Jülich - 52425 Jülich, Germany \\ M. Salerno \\ Dipartimento di Fisica "E.R. Caianiello" and \\ Istituto Nazionale di Fisica della Materia (INFM), \\ Universitá di Salerno, I-84081 Baronissi (SA), Italy
}

(Dated: 26th October 2018)

\begin{abstract}
A new class of models, generalizing Asymmetric Exclusion Process for many parallel interacting channels, is proposed. We couple the models with boundary reservoirs, study boundary-driven phase transitions and show that usually taken hydrodynamic description fails. The adequate hydrodynamic limit is then derived. We support our findings with Monte-Carlo simulations of the original stochastic system.

PACS numbers: 05.70.Ln, 64.60.Cn, 02.50.Ga
\end{abstract}




\section{INTRODUCTION}

Many interesting non-equilibrium physical phenomena (shock wave dynamics, boundary driven phase transitions, etc.) can be observed already in simplest models such as driven diffusing particle systems, recent review of which can be found e.g. in [1, 2]. These systems have a unique ability to feel the dynamics of the boundaries thanks to the presence of a flux of particles which brings information from boundaries to the bulk. In absence of a flux, indeed, boundaries would play marginal role, as in ordinary equilibrium statistical mechanics, but in presence of a flux the boundaries may dominate the bulk with the possibility to give rise to boundary driven phase transitions. These phenomena were indeed observed in one-species driven diffusive models [3, 4, 5] and in some models containing more than one species of particles (many-species models) 6, 7]. In a more general context, the problem was addressed in [4], where it was shown that the stationary state of systems with one species of particles with open boundaries in non-equilibrium depends only on the stationary flux. This implies that an extremum principle for the stationary flux can be formulated [5] and the properties of the stationary state can be obtained directly from the hydrodynamic limit. In this approach the stochastic model is mapped into a viscousless conservation law equation which is illdefined (the corresponding Cauchy problem admits multiple solutions). Since the dynamics of stochastic system (Markov process) is unique, the problem of regularization, i.e. how to single out the physical solution from a set of multiple solutions, arises. As is well known, this problem is resolved by adding a phenomenological small dissipative term (of order $\varepsilon$ ) to the conservation law equation and then taking the limit $\varepsilon \rightarrow 0$. For this purpose, a linear viscous term of the form $\varepsilon \partial^{2} u / \partial x^{2}$ is usually considered, although nonlinear terms of the type $\varepsilon f(u)$ $\partial^{2} u / \partial x^{2}$, with $f(u)$ being an arbitrary convex function, are also possible. For one-species systems this regularization procedure is quite robust in the sense that different choices of the viscosity term can be shown to lead to the same physical solution. For many-species models, however, this is not trivial. In this case the hydrodynamic limit is is described by a system of conservation laws (one for each particle species) and the regularization is usually achieved by adding a diagonal diffusion matrix of the form $\varepsilon \partial^{2} u^{i} / \partial x^{2}$, to the system. In spite of its simplicity, there is no evidence that this approach works also for these more complicated systems and the regularization problem of multi-species driven diffusive systems is still open.

The aim of the present Letter is two-fold. From one side, we introduce a new class of 
multi-species driven diffusive models which generalize the single chain asymmetric exclusion process (ASEP). These models possess nice properties such as product measure stationary state and particle-hole symmetry. From a physical point view, they describe the motion of particles in multi-channel cables with the particles in adjacent cables creating an effective barrier potential for the particles in a given cable to flow. From the other side, we study the hydrodynamic limit of these models by showing that the conventional regularization of the conservation law equations leads to wrong hydrodynamic results. General arguments which support this failure will be provided. On the contrary, we show that an alternative regularization, obtained directly from the microscopic dynamics, leads to correct results. A detailed comparison of the hydrodynamic predictions (both with conventional and alternative regularization) with direct Monte Carlo simulation of the original stochastic model confirms these results.

Although the mapping from hydrodynamic equations to stochastic processes is not unique, it is likely that our alternative regularization may work also for other multi-species driven diffusive models.

\section{THE MODEL}

To introduce the model, we consider $M \geq 2$ discrete chains on which particles can hop preferentially in one direction. The hopping between adjacent chains is forbidden but particles can move from a site $k$ to an empty site $k+1$ on the same chain with rates that depend on the particle configuration at adjacent sites of "neighboring" $S$ chains (the results will be qualitatively the same for other possibilities of next-neighboring hopping). Notice that such a dynamics does not satisfy detailed balance condition meaning that the system is far from thermodynamic equilibrium. The hopping rates for different configurations can be expressed in terms of a single parameter $\beta$ measuring the strength of the interaction between the chains. Let us denote with $r_{n}$ the hopping rate from site $k$ to site $k+1$ on the same chain, in presence of a total number $n$ of particles in adjacent chain sites. We restrict our search to the models having stationary product measures. In this case, calculation shows that the rates $r_{n}$ have to satisfy the condition $r_{n}-r_{n-1}=$ const for any $n$. Moreover, the rates $r_{n}$ turn out to depend only on $n$ and not on the particular configurations the $n$ particles

can assume. With $S$ chains-neighbors, any given pair of consecutive sites $k, k+1$ can have 

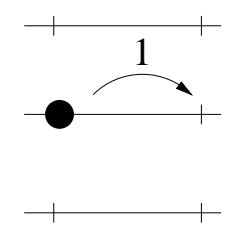
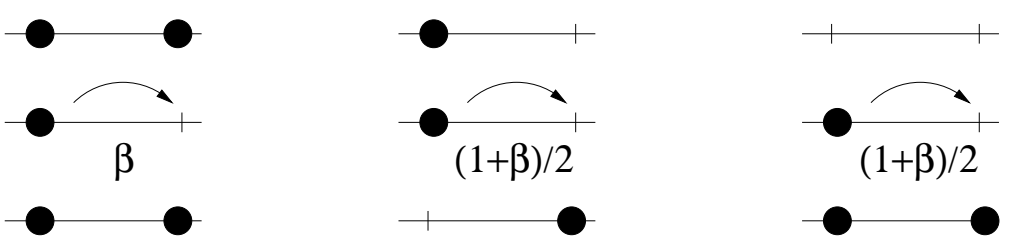

Figure 1: Elementary hopping processes happening in the case of $M=3, S=2$, with their rates. Four out of 16 possible configurations are shown. The rates (11) depend only on the total number of particles in the neighboring four sites.

from $n=0$ up to $n=2 S$ particles-neighbors. An example of the hopping process with the corresponding rates is shown in Fig. 1 for the case $S=2$. In general, for a chain having $S$ chains-neighbors [8], the rates of hopping (normalized to $r_{0}=1$ ) can be parametrized as

$$
r_{n}=1+n(\beta-1) / 2 S, \quad n=0,1, \ldots, 2 S .
$$

Notice that for $\beta=1$ the rates become independent on $n$ (i.e. the interchain interaction becomes zero) and the system splits into $M$ parallel uncoupled totally asymmetric exclusion processes (TASEP) 2, 9]. For $\beta<1$, the rates (11) monotonically decrease with number of particles-neighbors $n$, i.e. the presence of adjacent particles creates an effective barrier potential which slows down the particle movement.

The driven diffusive models described by (11) possess the following properties. i) Product measure. One can easily check that the model admits a stationary distribution in the class of product measures. This means that the stationary state of the model is spatially uncorrelated, both in the longitudinal and in the transverse direction. This property allows to obtain a simple analytic expression for the stationary flux (see below).

ii) Particle - hole symmetry. It is easy to check that an exchange of particles with holes plus the substitution $\beta \rightarrow 1 / \beta$ leaves the model invariant. Since parameter $\beta$ must be is in the range $0 \leq \beta<\infty$ (the rates in (11) are nonnegative), the present property allows to further restrict $\beta$ to the range $0 \leq \beta \leq 1$ only. In the limit of the strongest interaction $\beta=0$ the hopping of a particle in completely saturated environment becomes impossible $r_{2 S}=0$. Let us consider some specific examples.

Case $S=1$. In this case there is one "neighboring" chain for each given chain. This can happen if there are only two chains $(M=2)$ or if in the model with $M$ consecutive chains, the hopping along one chain depends only on the state of the next chain. Due to the property 
(i), the stationary flux on a given chain for $S=1$ can be computed straightforwardly as

$$
j(\rho, \sigma)=\rho(1-\rho)(1+(\beta-1) \sigma)
$$

where $\rho$ and $\sigma$ are average densities of particles on a given chain and on a chain "neighboring" to it, respectively. The case $M=2$ was considered in [10] where the properties of elementary excitations were studied.

Case $S=2$. In this case each pair of sites on two neighboring chains have up to 4 particleneighbors (see Fig. (1). this situation can be realized such a in the geometry of $M$ consecutive chains with periodic boundary conditions $M+1 \equiv 1$ in the transverse direction, where each chain $m$ has two chains-neighbors $m \pm 1$. The hopping rates are then obtained as

$$
r_{n}= \begin{cases}1 & \text { if } n=0 \\ (\beta+3) / 4 & \text { if } n=1 \\ (\beta+1) / 2 & \text { if } n=2 \\ (3 \beta+1) / 4 & \text { if } n=3 \\ \beta & \text { if } n=4\end{cases}
$$

The stationary flux on a chain $m$ in a system with average density $\rho_{k}$ on the chain $k$ can be obtained using property (i), as

$$
j_{m}=\rho_{m}\left(1-\rho_{m}\right)\left(1+\frac{1}{2}(\beta-1)\left(\rho_{m+1}+\rho_{m-1}\right)\right)
$$

Case $S>2$. For arbitrary number of $S$ chains-neighbors to a given one, the stationary flux is given by

$$
j_{m}=\rho_{m}\left(1-\rho_{m}\right)\left(1+\frac{1}{S}(\beta-1) \sum_{\text {neighbors }} \rho_{k}\right),
$$

where the sum is taken over the "neighboring" chains to a chain $m$, and $\rho_{k}$ is an average particle density on chain $k$.

From a physical point of view the case $S>2$ corresponds to a coaxial cable with many fibers, on which particles move, each fiber having $S$ fibers-neighbors surrounding it. A real system is always finite, so that boundary conditions, where particles can enter or exit the system must be imposed. To model boundaries, we couple model (II) with stationary particle reservoirs of densities $\rho_{L}^{m}$ on the left boundary of chain $m$, from where particles can be injected, and of the densities $\rho_{R}^{m}$, on the right boundary of it, where they can be 
extracted. The rates of extraction and injection are obtained from the boundary densities and for specific cases were given in [7, 11] .

To proceed further, we fix the total number of chains, $M$, the number of chains-neighbors of a given chain $S$, the right and left boundary densities $\rho_{R}^{m}, \rho_{L}^{m}$, and study bulk stationary densities that is, in the limit when time tends to infinity $t \rightarrow \infty$ [12], as the interchain interaction varies from $\beta=1$ (non interacting case) to $\beta=0$ (case of maximal interaction).

It is known that for an Asymmetric Exclusion process, which is a limit of our models for $\beta=1$, there is a first order non-equilibrium phase transition at $\rho_{R}^{m}=1-\rho_{L}^{m}>\frac{1}{2}$, where discontinuous transition from the low density phase $\rho_{\text {stat }}^{m}=\rho_{L}^{m}$ for $\rho_{R}^{m}=\left(1-\rho_{L}^{m}\right)-0$ to a high density phase $\rho_{\text {stat }}^{m}=\rho_{R}^{m}$ for $\rho_{R}^{m}=\left(1-\rho_{L}^{m}\right)+0$ happens. We will choose one of the boundary densities $\rho_{R}^{m}, \rho_{L}^{m}$ in the vicinity of this phase transition point (for $\beta=1$ ), different for different $m$ to exclude possible degeneracies, and look at the dependence of $\rho_{\text {stat }}^{m}(\beta)$ for different $M, S$. Alternatively, we shall look at the dynamic properties of the stochastic model and the hydrodynamic equation, comparing the time evolution of an initial state after certain time. The results of the Monte Carlo calculations can be seen on the graphs Figs 23 alongside with the results of numerical integration of corresponding partial differential equations arising from stochastic model in hydrodynamic limit. Derivation of these equations is given below.

\section{HYDRODYNAMIC LIMIT}

The naive continuum (Eulerian) limit of our stochastic dynamics on the lattice is a system of conservation laws

$$
\frac{\partial \rho^{Z}(x, t)}{\partial t}+\frac{\partial j^{Z}}{\partial x}=\epsilon \frac{\partial^{2} \rho^{Z}}{\partial x^{2}} ; \quad Z=1,2, \ldots, M
$$

with a phenomenological vanishing viscosity $\varepsilon \rightarrow 0$ (regularization term) on the right hand side added. Here $\rho^{Z}(x, t)$ denote coarse-grained particle densities while $j^{Z}$ — the particle fluxes given by Eq. (5). The regularization term in Eq. (60) leads to the correct answer

for the initial Riemann problem, as compared to the stochastic model (see 10]) but fails to describe the reflection from the boundaries (see [13], Figs 2, 3).

An adequate regularization is obtained by averaging exact lattice continuity equations of 


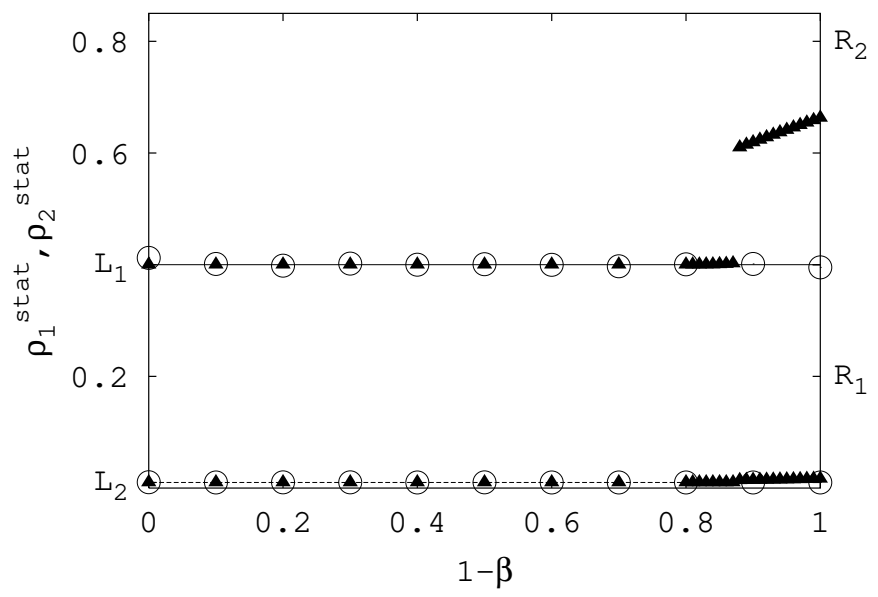

Figure 2: Comparison of predictions of MonteCarlo (open circles), the hydrodynamic equations Eq. (9) (line) and Eq.(6) (filled triangles) for stationary densities of particles in the system as a function of interchain interaction $\beta-1$. Parameters are: $M=2, S=1$, the boundary densities of the chains are $\rho_{L}^{1}, \rho_{L}^{2}=0.4,0.01$ on the left and $\rho_{R}^{1}, \rho_{R}^{2}=0.2,0.8$ on the right end.

the stochastic process

$$
\frac{\partial}{\partial t} \hat{n}_{k}=\hat{\jmath}_{k-1}-\hat{\jmath}_{k}
$$

for occupation number operators $\left\langle\hat{n}_{k}\right\rangle \rightarrow \rho(x, t)$ of any given chain, making the lattice constant infinitesimally small $k, k+1 \rightarrow x, x+a$. For the case (11), the local flux operator $\hat{\jmath}_{k}$ between the sites $k$ and $k+1$ reads (see [10] for a derivation of (8) in the case $M=2, S=1$ ):

$$
\hat{\jmath}_{k}=\hat{n}_{k}\left(1-\hat{n}_{k+1}\right)\left(1+\frac{\beta-1}{2 S} \sum_{p=1}^{S}\left(\hat{m}_{k}^{p}+\hat{m}_{k+1}^{p}\right)\right) .
$$

Here $\hat{m}_{k}^{p}, p=1, \ldots S$ are occupation number operators for a site $k$ of the chains neighboring to a given one. We substitute (8) into (7), average, factorize and Taylor expand the latter with respect to the lattice constant $a$ according to $\left\langle\hat{n}_{k+1}\right\rangle=\rho(x, t)+a \frac{\partial \rho}{\partial x}+\frac{a^{2}}{2} \frac{\partial^{2} \rho}{\partial x^{2}}+O\left(a^{3}\right)$, etc. Expanding the resulting equation in powers of $a$ and keeping terms up to the second order, we obtain the following hydrodynamic equation

$$
\frac{\partial \rho^{Z}(x, t)}{\partial t}+\frac{\partial j^{Z}}{\partial x}=\epsilon \frac{\partial}{\partial x}\left(\frac{\partial \rho^{Z}}{\partial x}\left(1+\frac{\beta-1}{S} \sum_{p=1}^{S} \rho^{p}\right)\right) ; \quad Z=1,2, \ldots M
$$

where $\rho^{p}=\rho^{p}(x, t)$ are local densities of the chains neighboring to a given chain $Z, \varepsilon=$ $\frac{a}{2} \rightarrow 0 ; \quad \frac{\partial}{\partial t} \rightarrow 2 \varepsilon \frac{\partial}{\partial t}$. Notice that Eq. (9) is valid for arbitrary $M, S$ and for $M=2, S=1$ 


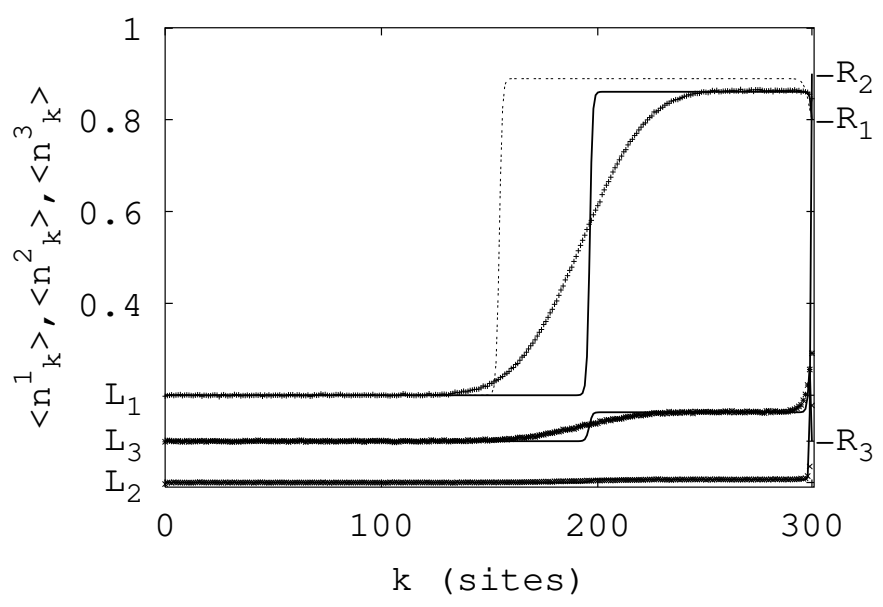

Figure 3: The time evolution of the stochastic model (11) with $\beta=0, M=3$. Comparison of average density profiles given by Monte-Carlo simulation (points), hydrodynamic equations (9) (lines), and naive viscosity approach (6) (broken line). The system has evolved for $t=1600$ Monte Carlo steps from homogeneous initial condition $\left\langle n_{k}^{Z}\right\rangle=\rho_{L}^{Z}$, matching the left boundary densities $\rho_{L}^{1}=0.2, \rho_{L}^{2}=0.01, \rho_{L}^{3}=0.1$. The corresponding right boundary particle densities are 0.8/0.9/0.1. An average over $10^{5}$ histories is taken. One sees that neither the position nor the level of the shock are predicted correctly by the naive viscosity approach (the broken line).

coincides with the hydrodynamic equation derived in Ref. [10].

To check the validity of this approach we have compared direct numerical integrations of (9) with Monte Carlo simulations of the original stochastic model described by the rates in Eq. (11). As a result we obtain that, while the agreement between the Monte Carlo predictions and the numerical integration of Eq. (9) is excellent, the comparison with the standard hydrodynamic limit gives rise to inconsistencies (see discussion below) both in the stationary regime and during relaxation towards stationarity. This is shown in Fig 2 for system of two and in Fig 3 for three chains with $S=1$. From these figures it is clear that the choice of the viscosity is indeed crucial to obtain correct results, since different choices will produce different answers. We checked that Eq. (99) gives results which coincide, up to non-universal boundary layer and interface width, with Monte Carlo simulations also for other values of $M, S$, and we believe this fact is valid in general.

The above analysis can be easily generalized to the case in which the hopping along the chains occurs in both directions. In this case the corresponding flux to the left is obtained 
from the one to the right by exchanging $\rho(1-\rho)$ in (5) with the term $(p-q) \rho(1-\rho)$, where $p$ and $q$ denote the particle hopping rates on the $m$-th chain to the right and to the left in the empty environment (the general conditions for a product measure state can be found in 14]).

It is interesting to discuss why a diagonal diffusion matrix leads to inconsistent results for multi-species systems. To this regard, we remark that one can formally obtain a diagonal diffusion matrix in Taylor expansion of (7) by adding to the flux (8) an additional term as, e.g.

$\hat{\jmath}_{k}=\hat{n}_{k}\left(1-\hat{n}_{k+1}\right)\left(1+\frac{\beta-1}{2 S} \sum_{p=1}^{S}\left(\hat{m}_{k}^{p}+\hat{m}_{k+1}^{p}\right)\right)+\left(\frac{\beta-1}{4 S}\left(\hat{n}_{k+1}-\hat{n}_{k}\right) \sum_{p=1}^{S}\left(\hat{m}_{k}^{p}+\hat{m}_{k+1}^{p}\right)\right)$.

If one substitutes the above expression into (7) and performs the same analysis as before, one obtains the resulting equation in the form (6). In this case, however, the factorization becomes invalid because the flux operator (10) describes different process with correlations in the stationary state and therefore with a stationary flux different from (5). Thus, by making the diffusion matrix diagonal one expects inconsistencies. This can be seen by considering a microscopic state with all but one chains completely filled with particles, the chain partially filled having an empty region separated by a completely filled one, $\hat{n}_{k}=0, k \leq 0$ and $\hat{n}_{k}=1$, $k>0$. According to the dynamic rules in Fig no movement is allowed, while from Eq. (17) (with averaged and factorized flux as in Eq. (10)), we obtain that $\frac{\partial}{\partial t} \hat{n}_{0} \neq 0$. Notice that although the choice of the second term in Eq. (10) is not unique, the above arguments for inconsistency would still be valid in presence of alternative choices. These inconsistencies are clearly seen on Fig 2, where a comparison between stationary densities of the two chain system $(M=2)$ obtained from Monte Carlo calculations and hydrodynamic equations (69) is made, as a function of the interchain interaction $1-\beta$. The naive description (6) shows a phase transition at $\beta \approx 0.1$, while Monte Carlo and (9) show no effect. The occurrence of a phase transition to high densities can be understood as follows. Since the right boundary density of particles of chain 2 is high $\left(\left(\rho_{2}\right)_{R}=0.8\right.$, it becomes more and more difficult for the particles of chain 1 to exit the chain with increasing interaction, so that "traffic jam" occurs at the exit, this leading to the phase transition. This effect is greatly overestimated by the naive hydrodynamic description ( 6) (in physical system the transition happens for higher left boundary density $\left.\left(\rho_{1}\right)_{L} \gtrsim 0.48\right)$. 


\section{CONCLUSION}

In conclusion, we have introduced a class of models which generalize the asymmetric simple exclusion process for an arbitrary number of chains and with hopping rates given as a function of the local configuration in the neighboring chains (11) describing the effective friction a particle encounters moving in a dense environment. For these models we derived the stationary flux, and modified the conventional choice of the viscosity term in the hydrodynamic limit (see, e.g., 15]) in order to get a good agreement with Monte Carlo simulations

of the original stochastic process. Although the problem of the hydrodynamic limit has been discussed on the specific example of the introduced models, the results are expected to be valid in general (the advantage of using our model is only for computational convenience since the product measure property allows to obtain the bulk flux, boundary conditions, etc., in an easy and straightforward manner.)

\section{Acknowledgments}

V.P. thanks G.M. Schütz for fruitful discussions, and the Department of Physics at the University of Salerno for hospitality. Financial support from the Deutsche Forschungsgemeinschaft, and from the three month grant of the Istituto Nazionale di Fisica della Materia (INFM) unitá di Salerno is gratefully acknowledged

[1] G.M. Schütz Exactly solvable models for many-body systems far from equilibrium, in: Phase Transitions and Critical Phenomena, C. Domb and J. Lebowitz (eds) (Academic, London, 2000 ) vol. 19.

[2] T. M. Liggett Stochastic interacting systems: contact, voter and exclusion processes (Springer, Grundlehren der Mathematischen Wissenschaften, 1999) vol. 324.

[3] J. Krug Phys. Rev. Lett. 67, 1882 (1991).

[4] A. B. Kolomeisky , G. M. Schütz , E. B. Kolomeisky and J. PStraley J. Phys. A 31, 6911 (1998).

[5] V. Popkov and G. M. Schütz Europhys. Lett. 48, 257 (1999). 
[6] M. R. Evans , D. P. Foster , C. Godrèche and D. Mukamel Phys. Rev. Lett. 74, 208 (1995) J. Stat. Phys. 80, 69 (1995).

[7] V. Popkov and I. Peschel Phys. Rev. E 64, 026126 (2001).

[8] Different chains may have different number of chains-neighbors (e.g., due to topological reasons). In this case, in order to have a product stationary state in the system, one should require $r_{n}-r_{n+1}=$ const for the rates of hopping along all the chains. Clearly, this condition is satisfied by (11)

[9] G. Schütz and E. Domany J. Stat. Phys. 72, 277 (1993); B. Derrida , M. R. Evans , V. Hakim and V. Pasquier J.Phys.A 26, 1493 (1993).

[10] V. Popkov and G. M. Schütz J Stat. Phys 112, 523 (2003).

[11] J. S. Hager , J. Krug, V. Popkov and G. M. Schütz, Phys. Rev. E 63, 056110 (2001).

[12] Note that there are cases when stationary state has complicated structure, e.g., spatial coexistence of structurally different regions 7]. However in the models (1) that we consider, bulk density is a good order parameter for a stationary state description.

[13] V. Popkov, in preparation

[14] B. Tóth and B. Valkó J Stat. Phys 112, 497 (2003).

[15] A. Bressan, Hyperbolic systems of conservation laws, Oxford University Press, 2000). 